\title{
The Antipsychotic Agent Sertindole Exhibited Antiproliferative Activities by Inhibiting the STAT3 Signaling Pathway in Human Gastric Cancer Cells
}

\author{
Chunyan Dai ${ }^{1}$, Pei Liu ${ }^{1}$, Xi Wang ${ }^{1}$, Yifei Yin ${ }^{1}$, Weiyang Jin², Li Shen ${ }^{3}$, Yuzong Chen ${ }^{4}$, Zhe Chen ${ }^{1}$, Yiping \\ Wang ${ }^{1,5} \bowtie$
}

1. Digestive Pathophysiology of Zhejiang Province, the First Affiliated Hospital of Zhejiang Chinese Medical University, 54 Youdian Road, Hangzhou, 310006 , China

2. College of Life and Environmental Sciences, Hangzhou Normal University, Hangzhou, 310006, China

3. Institute of Basic Theory of TCM, China Academy of Chinese Medical Sciences, Beijing 100700, China

4. Bioinformatics and Drug Design Group, Department of Pharmacy and Center for Computational Science and Engineering, National University of Singapore, 117543 , Singapore

5. Key Laboratory of Integrated Traditional Chinese and Western Medicine for Diagnosis and Treatment of Digestive System Tumor, the First Affiliated Hospital of Zhejiang Chinese Medical University,54 Youdian Road, Hangzhou, 310006, China

$\square$ Corresponding author: Yiping Wang, Zhejiang Key Laboratory of Gastro-Intestinal Pathophysiology, the First Affiliated Hospital of Zhejiang Chinese Medical University,54 Youdian Road, Hangzhou, 310006, China. Tel.: Tel: +86-571-8660280; Fax: +86-571-86620280; E-mail: ypwang@zcmu.edu.cn

(c) The author(s). This is an open access article distributed under the terms of the Creative Commons Attribution License (https://creativecommons.org/licenses/by/4.0/). See http://ivyspring.com/terms for full terms and conditions.

Received: 2019.03.13; Accepted: 2019.09.12; Published: 2020.01.01

\begin{abstract}
Gastric cancer (GC) is the third leading cause of cancer-related death. Although the therapeutic approaches have improved, the 5-year survival rate of GC patients after surgical resection remains low due to the high rates of metastasis and recurrence. Patients with schizophrenia have significantly lower incidences of cancer after long-term drug treatment, suggesting the potential or partially ameliorate the risk of cancer development of antipsychotic drugs. The goal of this study was to explore antipsychotic drugs with an optional effective therapy against gastric cellular carcinoma. We found that sertindole, an atypical antipsychotic, exhibited anti-tumor efficacy on human GC cells in vitro and in vivo. Moreover, sertindole in combination with cisplatin dramatically enhanced apoptosis-induction in GC cells. In addition, the pro-apoptotic effect of sertindole on GC might in part, involved in inhibition of STAT3 activation and downstream signals, including Mcll, surviving, c-Myc, cyclin DI. Collectively, these results suggested that sertindole could be a potential anticancer reagent and be an attractive therapeutic adjuvant for the treatment of human GC.
\end{abstract}

Key words: gastric cancer, sertindole, cell apoptosis, cisplatin

\section{Introduction}

Gastric cancer (GC) remains an important cancer worldwide. It is the fifth most frequently diagnosed cancer and the third leading cause of cancer mortality, with over 1,000,000 new cases in 2018 and an estimated 783,000 deaths (equating to 1 in every 12 deaths globally) ${ }^{[1]}$. The epidemiology data indicate that incidence rates are markedly elevated in Eastern Asia, for example, China, Mongolia, Japan and Korea, while the rates in Northern America and Northern Europe are generally low. Currently, the primary means of treatment is systemic chemotherapy, however, either low response rates or high toxicity are led to the disappointing results. Hence, it is critical to develop new drugs and novel strategies of GC therapeutics.

Recently, epidemiological observations have shown that schizophrenia patients after long-term drug treatment exhibited an overall reduced risk of cancer, which made efforts to reevaluate old drugs with a view to determining their potential as anticancer agents. Some antipsychotic agents like chlorpromazine, thioridazine, trifluoperazine and 
clozapine have shown anticancer activity [2-6]. Sertindole, a phenylindole derivative (Figure 1), is an oral antipsychotic drug available since 1996 for the treatment of schizophrenia in Europe [7]. Sertindole is a potent antagonist at dopamine D2, serotonin 5-HT2, and a1-adrenergic receptors with high affinity $[8,9]$. Sertindole was reported to induce autophagy and autophagy-associated cell death by ROS production in neuroblastoma cells [10]. Sertindole also caused breast cancer cell death through autophagy-associated apoptosis via the directly-binding inhibition of 5-HT6 [11]. However, mechanism of the anticancer effect of sertindole is largely unknown.

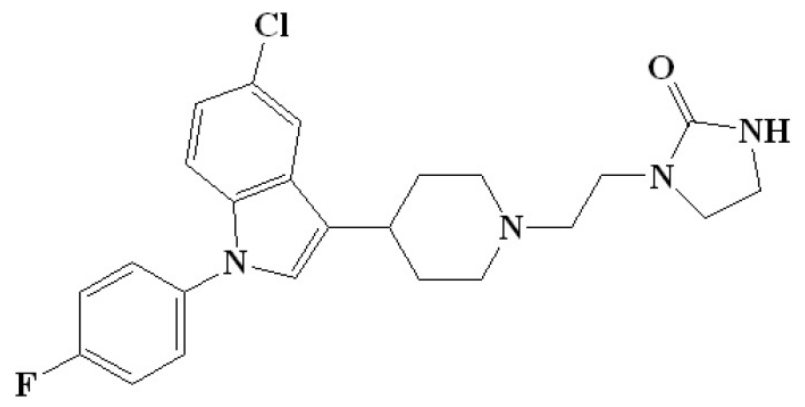

Figure 1. Chemical structure of sertindole.

Signal transducer and activator of transcription (STAT) proteins are involved in many biological responses and influence cell growth, survival, and metastasis [12-15]. Signal transducer and activator of transcription 3 (STAT3), the most strongly associated with tumorigenesis of STAT proteins, can be activated by a variety of upstream signals, including cytokines, growth factors and oncogenes. Generally, the recruited tyrosine kinase JAK can induce STAT3 phosphorylation at tyrosine 705, the phosphorylated STAT3s form self-dimerize and then shuttle to the nuclear for regulating the transcription of its targeting genes [16-17]. In contrast to the transient phosphorylation of STAT3 observed under physiological conditions of normal cells, STAT3 often persistently activates in many cancers and in many human cancer models [18]. Therefore, targeting inappropriate STAT3 activation is a promising therapeutic strategy. Several strategies have been investigated to target the STAT3 signaling pathway, including design of direct STAT3 small molecular inhibitors, inhibition of upstream tyrosine kinases, and the DNA binding complex [19-21]. For example, DU145 cells were observed that cell growth decreased and apoptosis increased when intracellular STAT3 protein levels were reduced by using antisense STAT3 oligonucleotides [22]. Akylating agent bendamustine stops the growth of cancer cells by binding to DNA and interfering with its replication through suppression on the SH2 domain of STAT3 [23]. In the stomach, STAT3 regulates diverse cellular functions including proliferation, basal homeostasis, acute inflammation, angiogenesis and apoptosis [24-25]. For instance, STAT3 was reported to constitutively activate in various human GC cells and its inhibition induced apoptosis [26].

In the present study, we investigated the anticancer effect of sertindole in GC. We observed that sertindole could suppress GC cells growth, induce cell apoptosis via suppressing phosphorylation of jak2stat3 and downstream targets. Oral administration of sertindole suppressed the tumor growth of GC in mice model. To the best of our knowledge, this the first report on the anticancer effects of sertindole targeting jak2-stat3 signaling pathway in GC.

\section{Materials and methods}

\section{Cell lines}

Human gastric cancer (GC) cell lines, including HGC27, MGC803, BGC823 and MNK45 cells (originally purchased from Cell Bank of the Chinese Academy of Science (Shanghai, China)), and the normal human hepatocyte cell line LO2 and human hepatic fetal epithelial cells WRL 68 cells (purchased from Cell Bank of the Chinese Academy of Science (Shanghai, China)) were maintained in RPMI-1640 medium(Gibco®, Hangzhou MultiSciences Biotech Co., Ltd., Hangzhou, China) supplemented with 10\% fetal bovine serum (Hyclone). All cells were incubated at $37^{\circ} \mathrm{C}$ in a humidified atmosphere containing $5 \%$ $\mathrm{CO}_{2}$.

\section{Reagents}

Sertindole (empirical formula, $\mathrm{C}_{24} \mathrm{H}_{26} \mathrm{ClFN}_{4} \mathrm{O}$; molecular weight, 440.94) was purchased from Sigma (USA). The sertindole was initially dissolved in dimethyl sulfoxide (DMSO) to obtain a $10 \mathrm{mM}$ stock solution and stored at $-20^{\circ} \mathrm{C}$, fresh dilutions in medium were made before use. Fetal bovine serum (FBS), $0.25 \%$ trypsin containing EDTA was obtained from Gibco (USA). Cell Counting Kit-8 (CCK-8) was purchased from DoJinDo (Japan).

\section{In vitro cytotoxicity}

The in vitro cytotoxicity of sertindole was measured by CCK- 8 , as described in the manufacturer's protocol. Briefly, $5 \times 10^{3}$ cells per well were plated in 96-well plates and treated with sertindole at various concentrations for $24 \mathrm{~h}$. Then, the medium with sertindole was replaced with $200 \mu \mathrm{L}$ of fresh medium along, $10 \mu \mathrm{L}$ CCK-8 solution was added into each well and incubated at $37{ }^{\circ} \mathrm{C}$ for $4 \mathrm{~h}$. Absorbance was measured at $450 \mathrm{~nm}$ using a spectrophotometer (Bio-Rad, USA). 


\section{Cell apoptosis assays}

The Annexin V-FITC Apoptosis Detection Kit (BD Biosciences) was used for apoptosis assays. Cells were seeded in 6-well plates at $2 \times 10^{5}$ per well and harvested after treated with or without sertindole for 24 hours, stained according to the manufacturer's protocol. The cells were analyzed with a FACStar flow cytometer (Canto II), and the data were analyzed using the MODFIT software (BD).

\section{Protein extraction and western blotting}

Total proteins were prepared from cultured cell samples by complete cell lysis (Roche) with protease and phosphatase inhibitors. Denatured proteins (20-50 ug) were separated on SDS-PAGE and transferred to membranes. The following primary antibodies were used: JAK2, p-JAK2, STAT3, pSTAT3 (Tyr 705), Mcl-1, Survivin, c-Myc, cyclin D1, PARP, Caspase 3, Caspase 9, XIAP, bcl 2, GAPDH, $\beta$-actin, tublin (all from Cell Signaling Technology). The bands were scanned using ChemiDocXRSt Imaging System (Bio-Rad).

\section{Tumor xenograft model}

Male BALB/c nu/nu nude mice at the age of 4-5 weeks (Zhejiang Academy of Medical Sciences), were housed at a specific pathogen-free environment in the Animal Laboratory Unit, Zhejiang Chinese Medical University, China. Mice were administered with sertindole $(10 \mathrm{mg} / \mathrm{kg}$ or $20 \mathrm{mg} / \mathrm{kg})$ or cisplatin $(5 \mathrm{mg} / \mathrm{kg})$ or equivalent concentration of DMSO every two days. All mice were sacrificed after 16 days. All procedures involving animals were conducted with the approved of the Committee on Animal Care in the First Affiliated Hospital of Zhejiang Chinese Medical University.

\section{Statistical analysis}

All numeric values are shown as mean \pm SD. Each set of experiment was repeated at least three times. Statistically significant differences between experimental and untreated control groups were detected using Student's unpaired t-tests. Difference was considered to be significant if $p<0.05$.

\section{Results}

\section{Sertindole suppresses proliferation of GC cells}

To evaluate the growth-suppressive effects of sertindole, we first performed the cytotoxicity assay in HGC27, MGC803, BGC823 and MNK45 cells. The cells were treated with varying concentrations of sertindole for 24 hours. Our results showed that treatment with increasing concentrations of sertindole significantly suppressed the growth of all the four GC cell lines in a concentration-dependent manner. The detailed IC50 values of sertindole against different cell lines were shown as table 1, the IC50 of sertindole after 24 hours treatment ranged from 4 to $16 \mu \mathrm{M}$ in all the four cell lines (Figure 2). In addition, we measure IC50 of sertindole against normal human hepatocyte cell line LO2 and human hepatic fetal epithelial cells WRL 68 cells. The IC50 values of LO2 and WRL 68 were $10.31 \pm 0.17$ and $12.71 \pm 0.33 \mu \mathrm{M}$, respectively. The IC50 curve of these two cell lines was shown as Supplementary figure 1. Compared to gastric cancer cells, the IC50 of the two normal hepatic cell lines was lower than BGC823 $(15.85 \pm 2.19 \mu \mathrm{M})$, but higher than the other three gastric cancer cell lines, suggesting that sertindole couldn't specifically inhibit the growth of gastric cancer cells. Collectively, these results suggested potential cytotoxic effects of sertindole in GC cells.

Table 1. IC 50 values of sertindole against different human gastric cancer cell lines.

\begin{tabular}{ll}
\hline Cell lines & IC $_{50}(\boldsymbol{\mu M})$ \\
\hline HGC27 & $9.75 \pm 2.62$ \\
MKN45 & $4.97 \pm 1.00$ \\
MGC803 & $6.83 \pm 1.85$ \\
BGC823 & $15.85 \pm 2.19$ \\
\hline
\end{tabular}
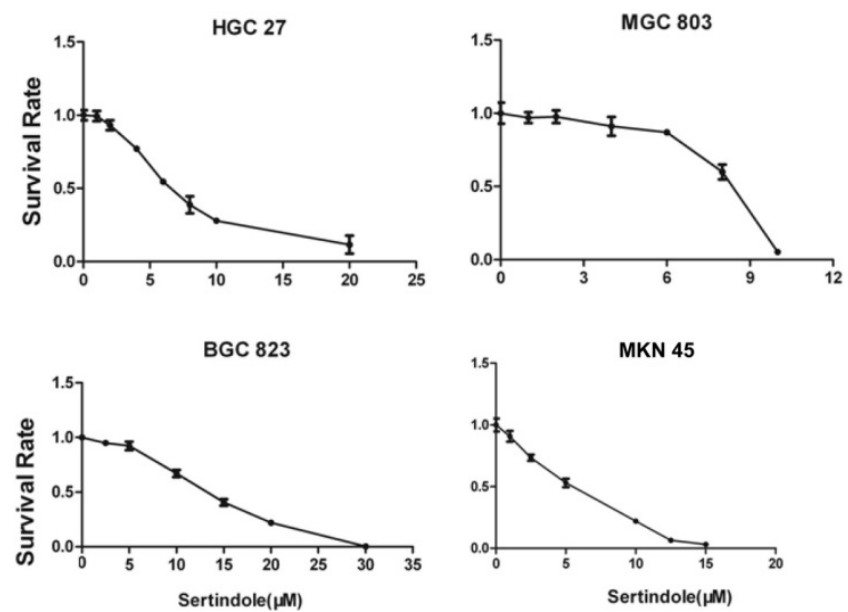

Figure 2. Sertindole inhibited gastric cancer (GC) cell proliferation in vitro. The growth inhibitory effect of sertindole was measured using the CCK-8 assay. Gastric cancer cell lines including HGC27, MGC803, BGC823 and MKN45 cells were treated with varying concentrations of sertindole (from 0 to $30 \mu \mathrm{M}$ for $24 \mathrm{~h}$ ). The experiments were performed in triplicate, and the data are presented as the mean \pm standard deviation (SD) of three separate experiments.

\section{Sertindole induced apoptosis of GC cells}

To determine the features of sertindole-induced GC cell growth inhibition, flow cytometric analysis was carried out in MGC803 and MNK45 GC cells for apoptosis analysis. Annexin V/PI staining was performed after GC cells were treated with different dosages of sertindole. Early and late apoptotic cells were counted. After 24 hours, sertindole-treated 
MGC803 and MNK45 GC cells exhibited a dose-dependent increase in annexin $\mathrm{V}$-positive cells compared with the control cells (Figure 3A-C). To investigate the apoptotic-associated signal transduction in GC cells after sertindole treatment, MGC803 and MNK45 GC cells treated with sertindole were analyzed with Western blot. As shown in Figure 3D, cleaved PARP, caspase-3 and caspase-9 was increased dose-dependently. These results indicated that sertindole induced apoptosis of GC cells.

\section{Sertindole inhibited constitutive JAK2-STAT3 phosphorylation in GC cells}

To elucidate the molecular mechanism of the growth-suppressive effects of sertindole, we carried out Western blot analysis of whole-cell lysates of MKN45 and MGC803 cells treated with 0, 5, 10 and 15 $\mu \mathrm{mol} / \mathrm{L}$ sertindole. As shown in Figure $4 \mathrm{~A}$, the treatment of sertindole $(8 \mathrm{hrs})$ significantly inhibited
STAT3-try 705 phosphorylation in MKN45 and MGC803 GC cells, and phosphorylation of JAK2 was significantly down-regulated in MGC803 GC cells. The inhibition on JAK2 and STAT3-try 705 phosphorylation was in a concentration-dependent manner. Moreover, we treated MGC803 and MKN45 cells with sertindole at $15 \mu \mathrm{mol} / \mathrm{L}$ for $0,0.5,1,2,4,8$ hours, the inhibition of STAT3-try 705 phosphorylation was observed in a time-dependent manner (Figure 4B). In addition, the levels of downstream targets of STAT3, including Mcl-1, survivin, c-Myc and cyclin D1, were obviously decreased by sertindole treatment. The inhibition of sertidole on these proteins was time- and dosages-dependent (Figure 4C). Taken together, these results indicated that sertindole suppressed GC cell survival by inhibiting the activation of JAK2-STAT3 signal pathway and expression of downstream targets.

A

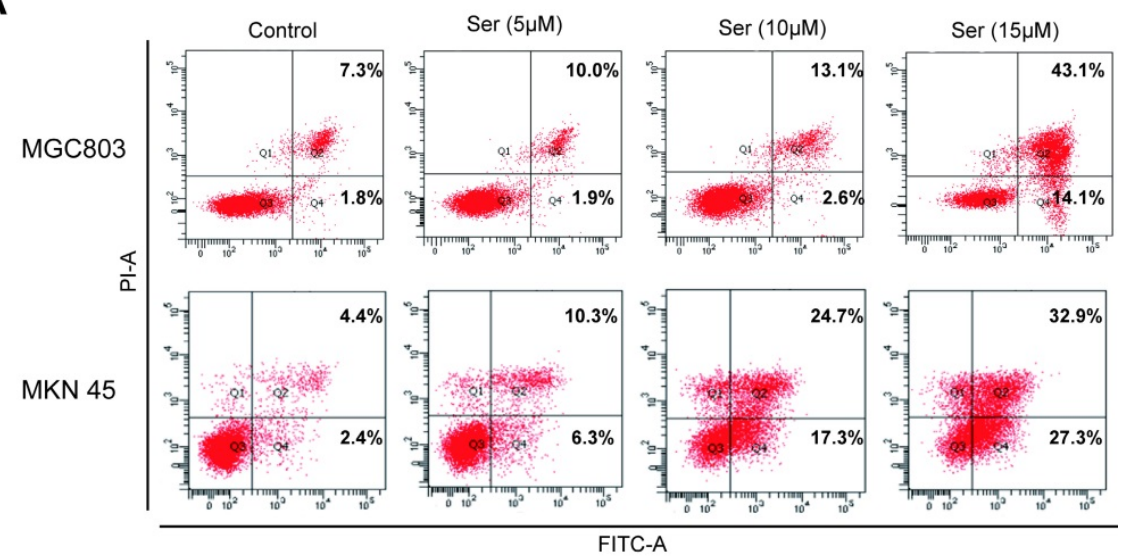

B

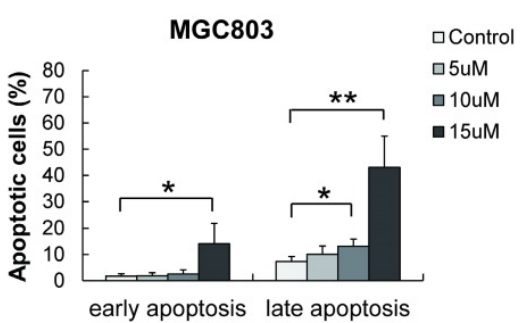

C

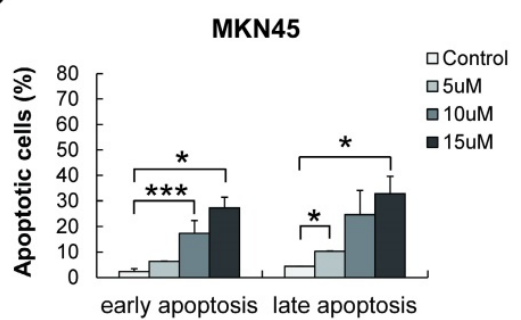

D

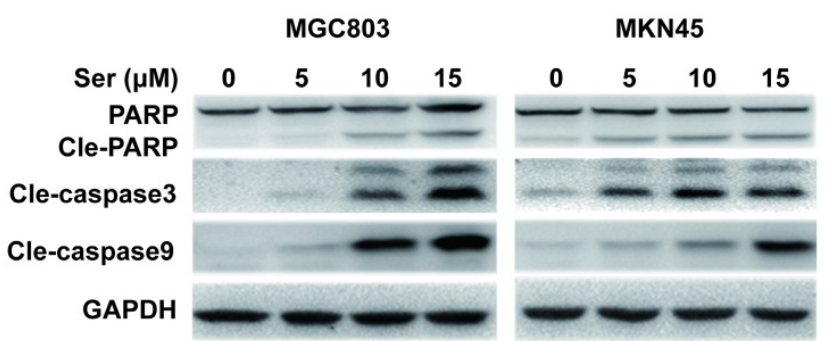

Figure 3. Sertindole induced cell apoptosis in GC cells. (A) MGC803 and MKN45 cells were treated with gradient concentrations of sertindole for 24 h. Then, the cells were stained with $\mathrm{Pl}$ at $37^{\circ} \mathrm{C}$ for $30 \mathrm{~min}$ and measured by flow cytometry after collection. (B) Quantitative analysis of apoptotic cells. The percentage of cells in different phases of cell apoptosis was represented by a bar diagram. Data are presented as the mean \pm SD of three independent experiments. (C) Effects of sertindole on the expression of apoptotic-associated proteins. (D) MGC803 and MKN45 GC cells were treated with $0,5,10$ and $15 \mu M$ of sertindole for $24 \mathrm{~h}$, and cell lysates were subjected to western blot analysis with cleaved PARP, caspase-3 and caspase- 9 antibodies. 


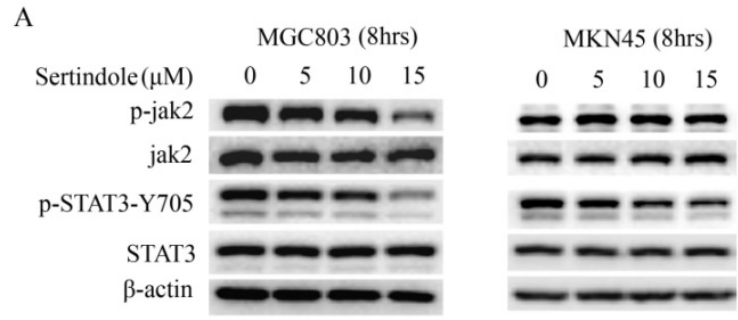

B

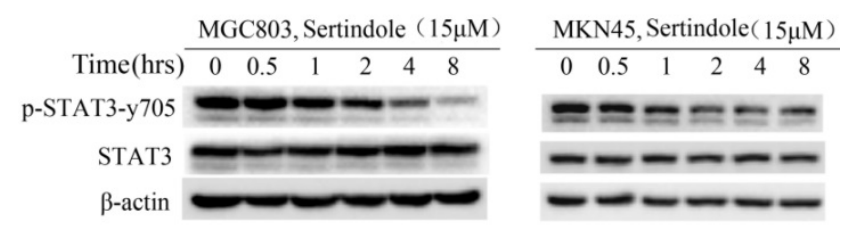

C

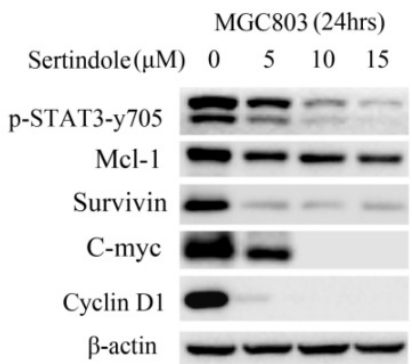

MGC803, Sertindole ( $15 \mu \mathrm{M}) \quad$ MKN45, Sertindole $(15 \mu \mathrm{M})$

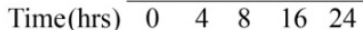

Mcl-1 $\longrightarrow-\infty$

Survivin

C-myc

Tublin

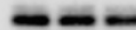

Figure 4. Sertindole suppressed GC cell proliferation through inhibition of JAK2-STAT3 signal pathway. (A) The GC cells were treated with sertindole at gradient concentrations for $8 \mathrm{~h}$. The cell lysates were then separated by $12 \%$ SDS-PAGE electrophoresis, and the levels of proteins related to JAK-STAT3 signaling pathway were detected. (B) MGC803 and MKN45 cells was treated with $15 \mu \mathrm{M}$ sertindole for $0,0.5,1,2,4$ and $8 \mathrm{~h}$, the levels of STAT3 and phosphorylated STAT3 (p-Stat3-y705) were detected by western blot analysis. (C) Western blot determined the levels of STAT3 downstream targets, including Mcl1, surviving, c-Myc, cyclin D1.

\section{Sertindole synergistically inhibits GC cells in vitro while combined with cisplatin}

To determine whether sertindole could sensitize GC cells toward chemotherapeutic agents, we treated MKN45 and MGC803 cells with sertindole or cisplatin alone, sertindole together with cisplatin for 24 hours, then analysis the percentage of survival cells by cck8 assay. The results showed that higher concemtration of sertindole or cisplatin alone led to lower cell survival rate, and the cell survival rate was significantly decreased after treatment of cells with combination of sertindole and cisplatin (Figure 5).

Next, we examined the combination of sertindole and ciaplatin on apoptotic cells by using flow cytometry. As shown in figure 6A-B, while treating with $5 \mu \mathrm{g} / \mathrm{ml}$ of cisplatin for 24 hours, the early and late apoptotic cells were $10.0 \%$ and $8.9 \%$, respectively, in MKN45 cells. The combined treatment of sertindole and cisplatin in MKN45 increased the early and late apoptotic cells to $20.7 \%$ and $26.9 \%$, respectively. The similar phenomenon was observed in MGC803 cells, the treatment of sertindole together with cisplatin obviously increased the percentage of apoptosis cells than the addition of sertindole or cisplatin alone, suggesting that the combined sertindole and cisplatin treatment provided a significantly higher cytotoxic effect than cisplatin treatment alone. Next, we examined the changes in molecules related to the cytotoxic effects by Western blot. After adding sertindole together with cisplatin, MKN45 cells showed a higher cleaved PARP, cleaved caspase- 3 and cleaved caspase- 9 activity as compared with cells with single drug. Moreover, the expression of proteins related to cell growth and anti-apoptosis, such as XIAP, bcl2, c-Myc and cyclinD1 were down-regulated (Figures 6C). Taken together, these results indicated that sertindole enhanced cisplatin-mediated GC cell cytotoxic effects and sensitized GC cells toward ciaplatin.

\section{Antitumor effects of sertindole in a xenograft GC model}

We then examined the ability of sertindole to reduce the growth of human GC cells subcutaneously implanted in nude mice. MGC803 GC cells were injected subcutaneously into the right flanks of the mice. One week after tumor cell injection, the animals were randomized into 4 treatment groups that tumor volumes were similar among the groups. The four groups of mice were received vehicle, sertindole alone (10 mg/kg/day, oral gavage), sertindole alone (20 $\mathrm{mg} / \mathrm{kg} /$ day, oral gavage), cisplatin alone (5 $\mathrm{mg} / \mathrm{kg} /$ day, oral gavage) treatment. Treatment began two weeks after implantation and continued for up to 16 days. Tumor diameters were measured every 2 days. Animals were killed 16 days after the initiation of treatment, and the diameters of the excised tumors were measured. Comparatively, mice that received sertindole alone $(20 \mathrm{mg} / \mathrm{kg} /$ day, oral gavage) showed significantly lower tumor burden than those that received vehicle or sertindole alone $(10 \mathrm{mg} / \mathrm{kg} /$ day, oral gavage). Sertindole $(20 \mathrm{mg} / \mathrm{kg} /$ day, oral gavage) alone-treated mice demonstrated a similar level of tumor burden as the cisplatin $(5 \mathrm{mg} / \mathrm{kg} /$ day, oral gavage) group (Figure 7). The nude mice treated with $20 \mathrm{mg} / \mathrm{kg}$ of sertindole had a bright coat, normal appetite and slightly lighter weight than the control group, but the body weight was the same as the cisplatin group (Supplementary figure 2). 
A

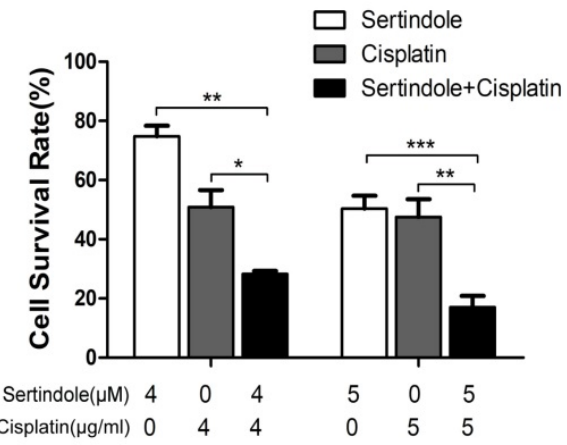

B

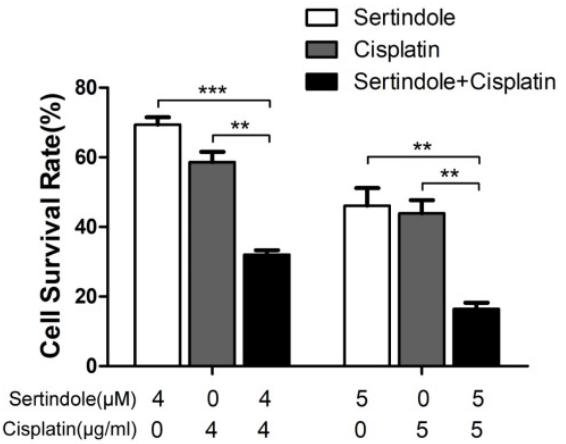

Figure 5. Combined treatment of sertindole and cisplatin to GC cells reduced cell viability. The effects of sertindole alone, cisplatin alone or sertindole together with cisplatin on cell viability were measured by cck8 assay. Combined treatment inhibited viability in MGC803 (A) and MKN45 (B) cell lines in a dose-dependent manner.

A

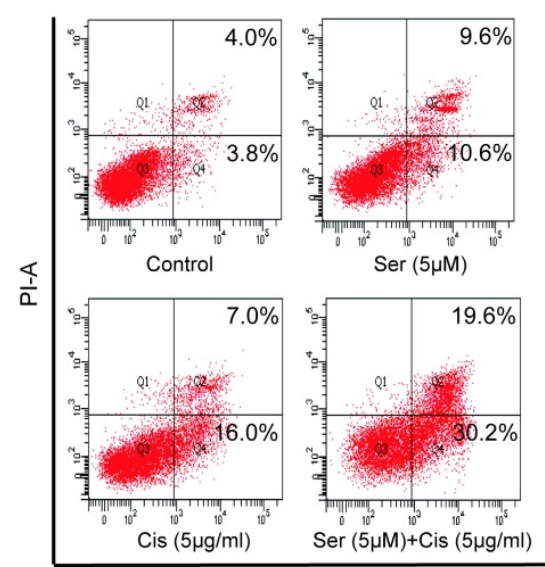

MGC803
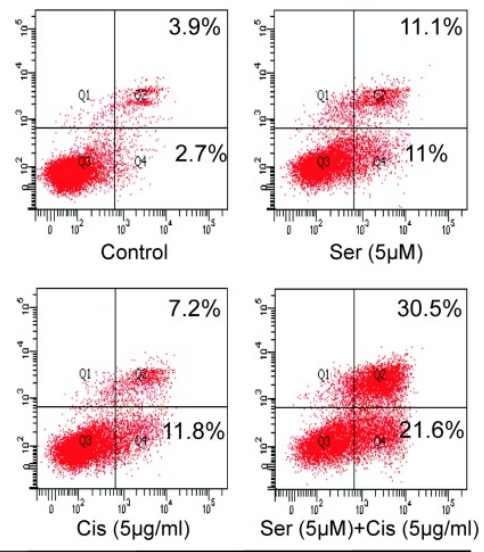

FITC-A

B
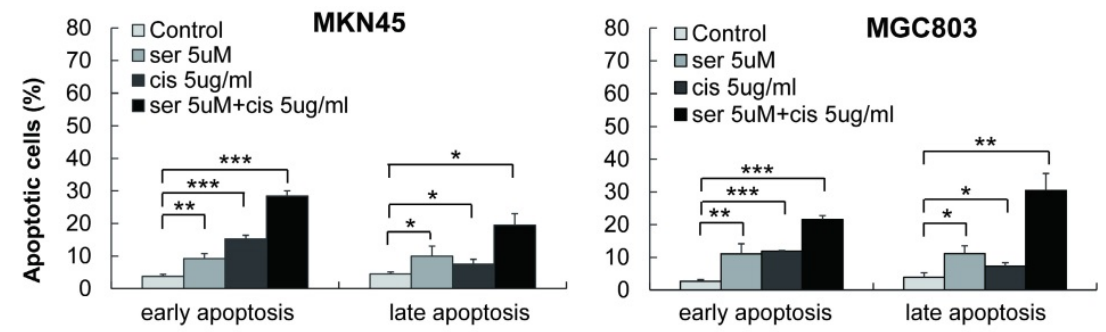

C

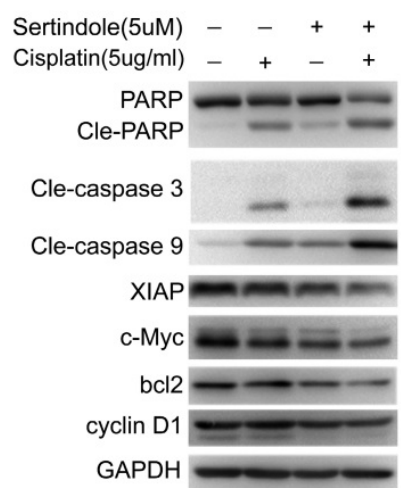

Figure 6. Sertindole potentiated the apoptotic-induction effects of cisplatin in GC cells. (A) The MKN45 and MGC803 cells were treated with sertindole alone, cisplatin alone or sertindole in combination with cisplatin for $24 \mathrm{~h}$. The cells were collected and stained with Pl at $37^{\circ} \mathrm{C}$ for 30 min, then measured by flow cytometry. (B) The histogram of cell apoptosis rates. Data are presented as the mean \pm SD of three independent experiments. (C) The MKN45 cells were treated with sertindole alone, cisplatin alone or sertindole in combination with cisplatin for $24 \mathrm{~h}$. The cell lysates were then separated by $12 \%$ SDS-PAGE electrophoresis, and cell apoptotic- and growth-related proteins expressions were detected by western blot analysis. 
A

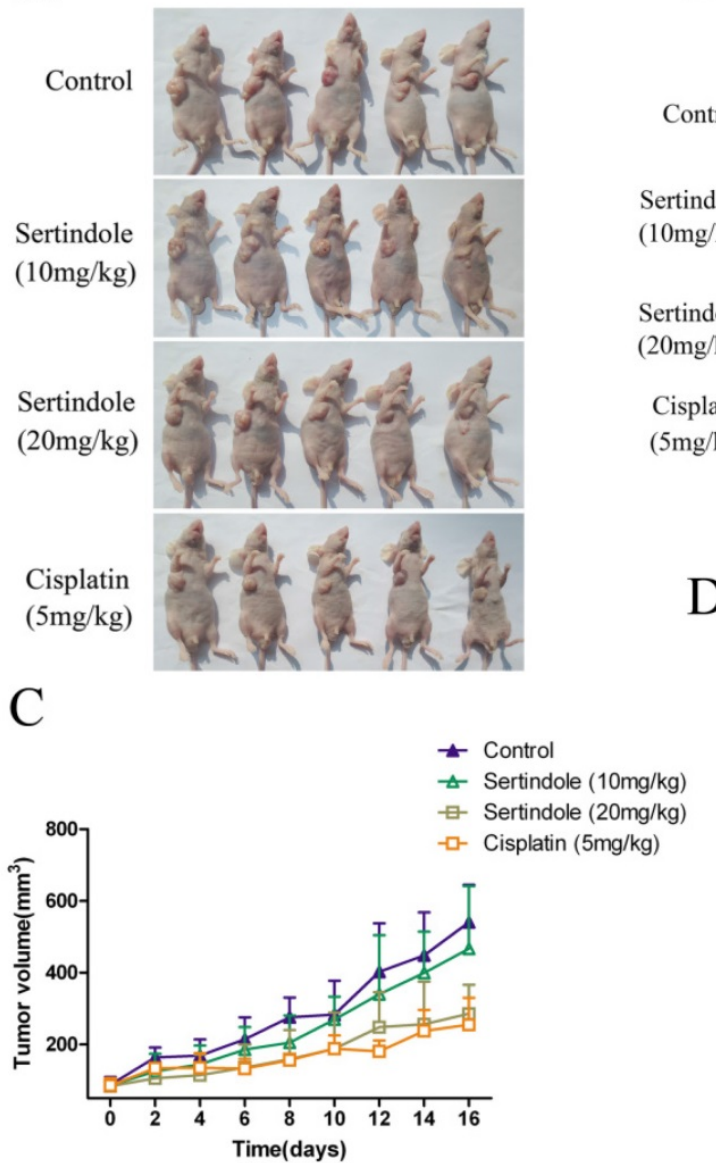

B

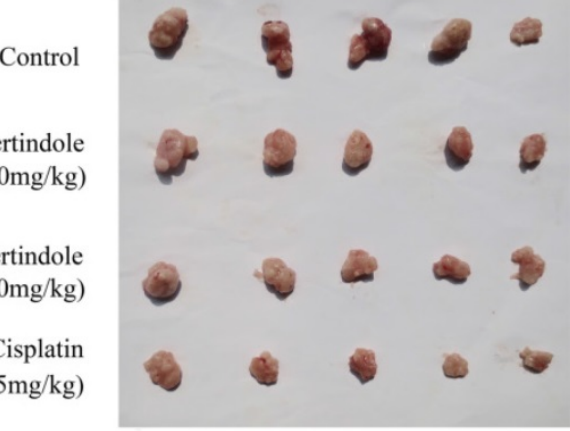

$\mathrm{D}$

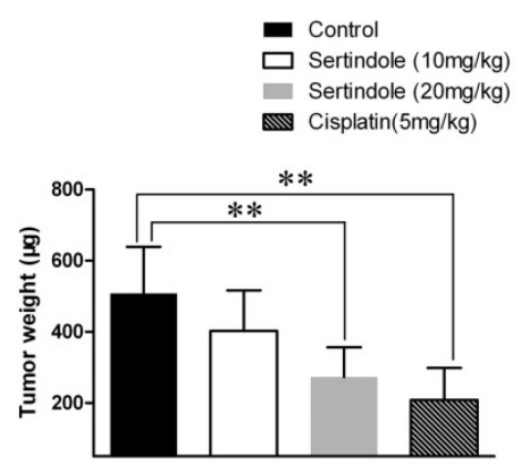

Figure 7. Sertindole inhibited tumor growth of MGC803 cells in nude mice. (A) The transplanted tumors in nude mice; (B) The transplantation tumors removed from nude mice; (C) Tumor volumes were measured on the indicated days; (D) The weight of the transplanted tumor. $* * P<0.01$ vs. control.

\section{Discussion}

Gastric cancer is the third leading cause of cancer-related death. Although the therapeutic approaches have improved, the 5-year survival rate of GC patients after surgical resection remains low due to the high rates of metastasis and recurrence. Moreover, the multidrug resistance is the other important reason for the failure of GC therapy. Recent years, reusing and repurposing of 'old' drugs is increasingly becoming an attractive strategy because of its lower overall development costs and shorter development timelines, as well as the use of de-risked compounds. The goal of this study was to explore old drugs with an optional effective therapy against gastric cellular carcinoma. We found that sertindole, an atypical antipsychotic, exhibited anti-tumor efficacy on human GC cells in vitro and in vivo. Moreover, sertindole in combination with cisplatin dramatically enhanced apoptosis-induction in GC cells. In addition, the pro-apoptotic effect of sertindole might involve in inhibition on activation of JAK2-STAT3 signaling pathway.
Antipsychotic drugs have been used for decades in various psychiatric clinical settings, epidemiological studies with various patient populations have demonstrated that patients with schizophrenia have significantly lower incidences of cancer than the general population, suggesting the potential or partially ameliorate the risk of cancer development of antipsychotic drugs. Trifluoperazine, an existing phenothiazine-like antipsychotic drug, was repurposed as a potential anti- cancer stem-like cell agent by overcoming epidermal growth factor receptor-tyrosine kinase inhibitor and chemotherapy resistance [3]. Penfluridol effectively reduces the growth of primary triple-negative breast cancer tumors and especially metastatic growth in the brain by inhibiting integrin signaling [27]. However, the underlying molecular mechanisms are yet to be elucidated. Sertindole is an atypical antipsychotic, which gives a lower incidence of extrapyramidal side effects and favorable metabolic profile at clinically effective doses than typical antipsychotic drugs [28-29]. After a reevaluation of its risks to cardiovascular safety and benefits to refractory patients, sertindole is 
available as a second-line choice for patients intolerant to other antipsychotic agents [30-31]. In the present study, we observed suppression effects of sertindole on GC cells growth with an $\mathrm{IC}_{50}$ concentration range from 4 to $16 \mu \mathrm{M}$, and oral administration at $20 \mathrm{mg} / \mathrm{kg}$ of sertindole suppressed the tumor growth of GC cells in mice model. Compared the administration of up to $40 \mathrm{mg} / \mathrm{kg}$ sertindole in rats, which the dose is 10 -fold greater than the maximal therapeutic dose of psychosis and does not elicit extrapyramidal symptom [32], the sensitivity of sertindole to GC cells was much higher. These results indicated that the potential anti-tumor effect of sertindole in GC.

Sertindole, a phenylindole derivative, is a potent and long acting compound as an antipsychotic drug. In vivo behavioural experiments showed that sertindole was dose-dependently binds to three receptors, which has the most pronounced effect on 5-HT2 receptors, lower effect on al-adreno receptors and the lowest effect on striatal D2 receptors on dopamine neurons in the rat [33]. Sertindole proved to be significantly better than first-generation drugs on relapse prevention. Moreover, sertindole was better than first-generation drugs refer to improve the life quality in schizophrenic patient [34]. A few papers explored the anti-cancer effects of sertindole [8-10], however, the underlying mechanism of the anti-tumour effect of sertindole remains less well characterised. In this study, we found that sertindole induced GC cell apoptosis, inhibited the phosphorylation of JAK2 and STAT3 at tyrosine 705 in time- and dose-dependent. STAT3 is in general transiently activated in normal cells but constitutively activated in a variety of solid tumors, including breast cancer, prostate cancer, head and neck cancer and GC cancers ${ }^{[35-38]}$. STAT3 is a key regulator of convergence of multiple oncogenic signaling pathways and is regarded as a "master regulator" that plays critical roles in human cell cycle, apoptosis, angiogenesis, stemness, metastasis to immune evasion [35]. In this study, the activation of STAT3 was suppressed when GC cells were exposed to sterindole, suggesting its therapeutic potential on GC. Further, the STAT3 has long been believed to act as a classic transcription factor to regulate the expression of a large number of target genes. For example, the protein encoded by the proto-oncogene c-Myc is a downstream target of STAT3 that is thought to be involved in tumor initiation and development. Activation of c-Myc is crucial for sustained tumour cell proliferation and survival [39-42], while suppression of c-Myc expression induces tumour regression in different tumour types [43] and promotes rapid tumour deterioration by triggering apoptosis or senescence ${ }^{[44]}$. In the present study, sertindole suppressed the expression of c-Myc in a dose-dependent manner. Taken together, sertindole could suppress the phosphorylation of STAT3 and c-Myc expression level, suggesting that the pro-apoptotic effect of sertindole on GC might in part, involve in inhibition of STAT3-c-Myc activation.

In conclusion, we showed that sertindole inhibited cell proliferation and induced cell apoptosis in GC cells. This event was associated with inhibition in JAK2-STAT3 signaling pathway and downstream signals, including Mcl1, surviving, c-Myc, cyclin D1. Moreover, sertindole exhibited anti-tumor efficacy on a xenograft GC model. In addition, sertindole in combination with cisplatin dramatically enhanced apoptosis-induction in GC cells. Combination therapy with sertindole and cisplatin might be a useful method to treat GC cells. All our data suggested that sertindole might be a promising candidate as an antitumor agent against GC.

\section{Supplementary Material}

Supplementary figures and tables.

http://www.jcancer.org/v11p0849s1.pdf

\section{Acknowledgments}

This work was funded by the National Natural Science Foundation of China (Grant No. 81473389, 81773945, 81703753, 81503297, 81803776, 81803775, 81873276); Natural Science Foundation of Zhejiang Province (Grant No. LQ18H280005); Beijing Municipal Natural Science Foundation (Grant No. 7172147); National Natural Science Foundation for Young Scientists of China (Grant No. 81403199); Fundamental Research Funds for the Central Public Welfare Research Institutes (Grant No. YZ-1856); Opening Project of Zhejiang Provincial First-rate Subject (Integrated Traditional and Western Medicine), Zhejiang Chinese Medical University (Grant No. ZXYJH2018003); Zhejiang Provincial Medical and Healthy Science Foundation of China (Grant No. 2020KY660).

\section{Author Contributions}

Data curation, Chunyan Dai, Pei Liu and Weiyang Jin; Formal analysis, Chunyan Dai, Xi Wang and Yiping Wang; Funding acquisition, Chunyan Dai, Pei Liu, Yifei Yin, Li Shen, Zhe Chen and Yiping Wang; Investigation, Chunyan Dai, Pei Liu, Weiyang Jin and Yifei Yin; Project administration, Zhe Chen and Yiping Wang; Writing - original draft, Chunyan Dai and Yiping Wang; Writing - review \& editing, Yuzong Chen, Zhe Chen and Yiping Wang.

\section{Competing Interests}

The authors have declared that no competing 


\section{interest exists.}

\section{References}

1. Bray F, Ferlay J, Soerjomataram I, et al. Global cancer statistics 2018: GLOBOCAN estimates of incidence and mortality worldwide for 36 cancers in 185 countries. CA Cancer J Clin. 2018; 68: 394-424.

2. Yin YC, Lin CC, Chen TT, et al. Clozapine induces autophagic cell death in non-small cell lung cancer cells. Cell Physiol Biochem. 2015; 35: 945-56.

3. Yeh CT, Wu AT, Chang PM, et al. Trifluoperazine, an antipsychotic agent, inhibits cancer stem cell growth and overcomes drug resistance of lung cancer. Am J Respir Crit Care Med. 2012; 186: 1180-8.

4. Shin SY, Lee KS, Choi YK, et al. The antipsychotic agent chlorpromazine induces autophagic cell death by inhibiting the Akt/mTOR pathway in human U-87MG glioma cells. Carcinogenesis 2013; 34: 2080-9.

5. Cheng HW, Liang YH, Kuo YL, et al. Identification of thioridazine, an antipsychotic drug, as an antiglioblastoma and anticancer stem cell agent using public gene expression data. Cell Death Dis. 2015; 6: e1753.

6. Chou FH, Tsai KY, Su CY, et al. The incidence and relative risk factors for developing cancer among patients with schizophrenia: a nine-year follow-up study. Schizophr Res. 2011; 129: 97-103.

7. Moore N, Hall G, Sturkenboom M, et al. Biases affecting the proportional reporting ratio (PPR) in spontaneous reports pharmacovigilance databases: the example of sertindole. Pharmacoepidemiol Drug Saf. 2003; 12: 271-81.

8. Spina E, Zoccali R. Sertindole: pharmacological and clinical profile and role in the treatment of schizophrenia. Expert Opin Drug Metab Toxicol. 2008; 4: 629-38.

9. Kasper S, Möller HJ, Hale A. The European post-marketing observational sertindole study: an investigation of the safety of antipsychotic drug treatment. Eur. Arch. Psychiatry Clin. Neurosci. 2010; 260: 59-68.

10. Shin JH, Park SJ, Kim ES, et al. Sertindole, a potent antagonist at dopamine $\mathrm{D}_{2}$ receptors, induces autophagy by increasing reactive oxygen species in SH-SY5Y neuroblastoma cells. Biol Pharm Bull. 2012; 35: 1069-75.

11. Zhang W, Zhang C, Liu F, et al. Antiproliferative activities of the second-generation antipsychotic drug sertindole against breast cancers with a potential application for treatment of breast-to-brain metastases. Sci Rep. 2018; 8: 15753.

12. Pastuszak-Lewandoska D, Domańska-Senderowska D, Kordiak J, et al Immunoexpression analysis of selected JAK/STAT pathway molecules in patients with non- small-cell lung cancer. Pol Arch Intern Med. 2017; 127: 758-764.

13. Amani H, Ajami M, Nasseri Maleki S, et al. Targeting signal transducers and activators of transcription (STAT) in human cancer by dietary polyphenolic antioxidants. Biochimie. 2017; 142: 63-79.

14. Wang S, Yu L, Shi W, et al. Prognostic roles of signal transducers and activators of transcription family in human breast cancer. Biosci Rep. 2018; 38(6). BSR20171175.

15. Chen L, Zhou D, Liu Z et al. Combination of gemcitabine and erlotinib inhibits recurrent pancreatic cancer growth in mice via the JAK-STAT pathway. Oncol Rep. 2018; 39: 1081-1089.

16. Sgrignani J, Garofalo M, Matkovic M, et al. Structural Biology of STAT3 and Its Implications for Anticancer Therapies Development. Int J Mol Sci. 2018; 19(6). E1591.

17. Masciocchi D, Gelain A, Villa S, et al. Signal transducer and activator of transcription 3 (STAT3): a promising target for anticancer therapy. Future Med Chem. 2011; 3: 567-97.

18. Yu H, Lee H, Herrmann A, et al. Revisiting STAT3 signalling in cancer: new and unexpected biological functions. Nat Rev Cancer. 2014; 14: 736-46.

19. Siveen KS, Sikka S, Surana R, et al. Targeting the STAT3 signaling pathway in cancer: role of synthetic and natural inhibitors. Biochim Biophys Acta. 2014; 1845: 136-54.

20. Yue P, Turkson J. Targeting STAT3 in cancer: how successful are we? Expert Opin Investig Drugs. 2009; 18:45-56.

21. Chai EZ, Shanmugam MK, Arfuso F, et al. Targeting transcription factor STAT3 for cancer prevention and therapy. Pharmacol Ther. 2016; 162: 86-97.

22. Mora LB, Buettner R, Seigne J, et al. Constitutive activation of Stat3 in human prostate tumors and cell lines: direct inhibition of Stat 3 signaling induces apoptosis of prostate cancer cells. Cancer Res. 2002; 62: 6659-6666.

23. Iwamoto $\mathrm{K}$, Uehara $\mathrm{Y}$, Inoue $\mathrm{Y}$, et al. Inhibition of STAT3 by anticancer drug Bendamustine. PLoS One. 2017; 12:e170709.

24. Wake MS, Watson CJ. STAT3 the oncogene-still eluding therapy? FEBS J. 2015; 282:2600-11.

25. Menheniott TR, Judd LM, Giraud AS. STAT3: a critical component in the response to Helicobacter pylori infection. Cell Microbiol. 2015; 17: 1570-82.

26. Kanda N, Seno H, Konda $\mathrm{Y}$, et al. STAT3 is constitutively activated and supports cell survival in association with survivin expression in gastric cancer cells. Oncogene. 2004; 23: 4921-9.

27. Ranjan A, Gupta P, Srivastava SK. Penfluridol: An Antipsychotic Agent Suppresses Metastatic Tumor Growth in Triple-Negative Breast Cancer by Inhibiting Integrin Signaling Axis. Cancer Res. 2016; 76: 877-90.

28. Van Kammen DP, McEvoy JP, Targum SD, et al. A randomized, controlled, dose-ranging trial of sertindole in patients with schizophrenia. Psychopharmacology (Berl). 1996; 124: 168-75.
29. Kane JM. Sertindole: a review of clinical efficacy. Int Clin Psychopharmacol. 1998; 13 (Suppl 3): S59-63.

30. Kane JM, Potkin SG, Daniel DG, et al. A double-blind, randomized study comparing the efficacy and safety of sertindole and risperidone in patients with treatment-resistant schizophrenia. J Clin Psychiatry. 2011; 72: 194-204.

31. Leucht S, Cipriani A, Spineli L, et al. Comparative efficacy and tolerability of 15 antipsychotic drugs in schizophrenia: a multiple-treatments meta-analysis. Lancet. 2013; 382: 951-962.

32. Ninan I, Kulkarni SK. Quinpirole, 8-OH-DPAT and ketanserin modulate catalepsy induced by high doses of atypical antipsychotics. Methods Find Exp Clin Pharmacol. 1999; 21: 603-8.

33. Skarsfeldt T, Perregaard J. Sertindole, a new neuroleptic with extreme selectivity on A10 and A9 dopamine neurones in the rat. Eur J Pharmacol. 1990; 182: 613-4.

34. Leucht S, Corves C, Arbter D, et al. Second generation versus first-generation antipsychotic drugs for schizophrenia: a meta-analysis. Lancet. 2009; 373: 31-41.

35. Banerjee $\mathrm{K}$, Resat $\mathrm{H}$. Constitutive activation of STAT3 in breast cancer cells: A review. Int J Cancer. 2016; 138: 2570-2578.

36. Kanda N, Seno H, Konda $\mathrm{Y}$, et al. STAT3 is constitutively activated and supports cell survival in association with survivin expression in gastric cancer cells. Oncogene. 2004; 23: 4921-4929.

37. Corvinus FM, Orth C, Moriggl R, et al. Persistent STAT3 activation in colon cancer is associated with enhanced cell proliferation and tumor growth. Neoplasia. 2005; 7: 545-555.

38. Scholz A, Heinze S, Detjen KM, et al. Activated signal transducer and activator of transcription 3 (STAT3) supports the malignant phenotype of human pancreatic cancer. Gastroenterology. 2003; 125: 891-905.

39. Felsher DW, Bishop JM. Reversible tumorigenesis by MYC in hematopoietic lineages. Mol Cell. 1999; 4:199-207.

40. Jain M, Arvanitis C, Chu K, et al. Sustained loss of a neoplastic phenotype by brief inactivation of MYC. Science. 2002; 297:102-104.

41. Marinkovic D, Marinkovic T, Mahr B, et al. Reversible lymphomagenesis in conditionally c-MYC expressing mice. Int J Cancer. 2004; 110: 336-42.

42. Pelengaris S, Littlewood T, Khan M, et al. Reversible activation of c-Myc in skin: induction of a complex neoplastic phenotype by a single oncogenic lesion. Mol Cell. 1999; 3: 565-77.

43. Wu CH, van Riggelen J, Yetil A, et al. Cellular senescence is an important mechanism of tumor regression upon c-Myc inactivation. Proc Natl Acad Sci USA. 2007; 104:13028-13033.

44. Soucek L, Whitfield J, Martins CP, et al. Modelling Myc inhibition as a cancer therapy. Nature. 2008; 455: 679-83. 